\title{
La Validité des Élévations Open-Source pour Différentes Échelles de Cartes Topographiques et Applications Géomatiques
}

\author{
Gomaa Mohammed Dawod* \\ Ibrahim Elsayed Ascoura**
}

\begin{abstract}
RÉSUMÉ
Cet article présente une analyse de quatre modèles d'élévation numériques mondiaux (MNE) open source et les compare sur deux profils topographiques (régions presque plates et collines) pour des applications en cartographie et en géomatique. L'objectif principal est de vérifier si les hauteurs, les intervalles de courbes de niveaux, les pentes et les profils topographiques basés sur les MNE sont valides pour toutes les échelles de la cartographie topographique, ce qui constitue un problème majeur dans les activités de cartographie. Deux études de cas, le delta du Nil en Égypte et la ville de La Mecque en Arabie Saoudite, ont été utilisées pour représenter des sites à topographie plate et modérée. Les MNE mondiaux étudiés incluent les modèles les plus récents: ASTER v.3, ACE 2, SRTMGL1 v.3, et NASADEM_HGT v.1 publiés en 2019 et 2020 avec des résolutions spatiales de 1 et 3 secondes d'arc. Les points de contrôle au sol précis disponibles (Ground Control Points, $G C P$ ) comprennent 540 stations dans le delta du Nil et 175 stations à La Mecque. Sur la base des ensembles de données disponibles dans deux zones d'étude, il a été constaté que la précision des MNE étudiés sur les points de contrôle connus varie de \pm 2.5 à \pm 5.1 mètres dans la région du delta du Nil, alors qu'elle varie entre \pm 5.1 et \pm 8.0 mètres dans la zone de La Mecque. Cela indique que l'utilisation des MNE dans la cartographie topographique diffère considérablement entre les régions plates et les zones vallonnées. Par conséquent, il est recommandé d'éviter d'utiliser les MNE pour développer des cartes topographiques à l'échelle 1:25000 ou plus dans les régions plates et à l'échelle 1:50000 ou plus dans les régions vallonnées. De plus, les résultats obtenus ont montré que toutes les pentes basées sur les GDEM ne correspondent pas aux pentes réelles du GCP connu sur une longueur de section allant jusqu'à 30 kilomètres. Ainsi, il est conclu que les MNE ne sont pas une source appropriée de données altimétriques pour la cartographie topographique à moyenne et grande échelle, et ne devraient pas être utilisés pour le profilage topographique dans des applications d'ingénierie et de géomatique précises. [Bul. Soc. Géog. d'Égypte, 2021, 94: 1-23]
\end{abstract}

Mots-clés: Carte topographique, MNE, altitudes, échelles cartographiques, intervalle de courbes de niveaux.

* Professeur, Institut de Recherche d'Arpentage, Centre National de Recherche sur l'Eau, Gizeh, Égypte.

* Professeur Assistant, Département de Géographie, Faculté des Sociales Sciences, Université d'Umm Al Qura, KSA.

For Correspondence: e-mail: ieascoura@uqu.edu.sa 


\section{Introduction}

Les cartes topographiques représentent un type principal de cartes utilisées dans de nombreuses applications géomatiques, telles que les projets d'ingénierie, la modélisation hydrologique, l'évaluation des risques de catastrophe, l'analyse géomorphologique et hydrogéologique et les applications environnementales. Les courbes de niveau représentent une information vitale apparaissant sur les cartes topographiques imprimées et numériques. Elles représentent les variations topographiques du terrain et permettent d'estimer les altitudes des points aléatoires requis. De nos jours, la forme la plus fréquente de stockage et d'analyse des ensembles de données altimétriques est le modèle numérique d'élévation (MNE). Il est bien connu que les hauteurs utilisées pour générer les courbes de niveau peuvent être obtenues à partir d'un large éventail de sources, notamment des levés terrestres, des données des systèmes mondiaux de navigation par satellite (Global Navigation Satellite Systems, GNSS), des photographies aériennes, des images satellite et des MNE. En outre, les systèmes d'information géographique (SIG) permettent la manipulation, le traitement et l'analyse des MNE pour générer de nombreux dérivés (outputs) pour la cartographie topographique du terrain, dont la génération de courbes de niveau pour une large gamme d'applications. Certaines de ces applications comprennent la production de cartes des risques d'inondation (Ogania et al., 2019), la cartographie des linéaments et structures géologiques (Seleem, 2013), l'analyse du paysage fluvial dans les régions montagneuses (Boulton et Stokes, 2018), le suivi des impacts de l'élévation du niveau de la mer (Hasan et al., 2015), la production de cartes topographiques (Ouerghi et al., 2015) et la modélisation du géoïde (Al-Karagy et Dawod, 2021).

Dans de nombreux pays en développement, des modèles MNE nationaux précis peuvent exister mais ne sont pas accessibles pour un usage public. Ainsi, les chercheurs utilisent généralement les MNE globaux gratuits open-source disponibles. Au cours des dernières décennies, de nombreux types de MNE, avec des couvertures et des résolutions spatiales variables, ont été produits et rendus librement accessibles sur des sites Web. Parmi ces MNE on peut citer: Shuttle Radar Topography Mission (SRTM), Advanced Spaceborne Thermal Emission and Reflection Radiometer (ASTER), EarthEnv-DEM90, Global Multi-resolution Terrain Elevation Data 2010 (GMTED2010), Global Land One-km Base Elevation (GLOBE), Global 30 secondes d'arc (GTOPO30) et Advanced Land Observing Satellite (ALOS)-based World 3D - 30 m (AW3D30).

La précision des MNE open source joue un rôle essentiel dans leur utilisation pour la cartographie et les études environnementales. Ainsi, de nombreuses études et recherches se sont concentrées sur l'estimation de la précision et de l'exactitude des MNE. Dawod et Al-Ghamdi (2017) ont 
examiné la fiabilité de huit MNE globaux pour des applications géomatiques en Égypte et en Arabie Saoudite. Les résultats ont montré que leur précision, en termes d'écart type sur les points de contrôle, est comprise entre \pm 1.9 et \pm 6.6 mètres. Des résultats similaires ont été rapportés dans d'autres régions telles que les États-Unis (Kulp et Strauss, 2016), l'Inde (Patel et al., 2016) et la Chine (Hu et al., 2017).

Plutôt que les cartes topographiques imprimées ou numériques, plusieurs applications Géomatiques notamment, utilisant les SIG, reposent sur une représentation numérique de la topographie. De telles activités nécessitent un niveau de précision altimétrique en fonction de leurs objectifs et de l'étendue spatiale de la zone d'étude. L'utilisation de MNE peut ne pas convenir aux applications géomatiques de haute précision. L'impact de l'élévation du niveau de la mer (Sea Level Rise, SLR) dans des régions petites ou modérées en est un exemple typique. On sait que le SRL moyen anticipé sur une base globale en 2081-2100 est estimé entre 0.40 et 0.63 mètre par rapport au niveau 1986-2005 (Church et al., 2013). La surveillance et l'estimation des impacts des risques d'une si petite élévation du niveau de la mer nécessitent une représentation du terrain de haute précision. Abdel Aziz et al. (2020) ont étudié la fiabilité de quelques MNE dans la délimitation des régions inondées en cas d'élévation du niveau de la mer dans la région du delta du Nil. Il a été constaté que la fiabilité de trois modèles globaux étudiés, par rapport à un modèle numérique de terrain (MNT) local de haute précision, est inférieure à 20\%. Des résultats similaires ont été rapportés par d'autres chercheurs dans d'autres régions (Sande et al., 2012). Un autre exemple est l'utilisation de MNE de faible précision dans les applications d'ingénierie. Zhang et Chu (2015) ont souligné que la quantification précise du stockage de la dépression de surface dépend de manière significative des caractéristiques topographiques basées sur le modèle numérique d'élévation MNT, principalement de la précision verticale et de la résolution spatiale. D'autre part, il existe certaines applications géomatiques qui pourraient être réalisées en utilisant la précision de quelques mètres des MNE. La reconnaissance, la planification de projets pour des régions spatiales moyennes ou grandes, la modélisation hydrologique, l'estimation du ruissellement de surface et la modélisation du géoïde pourraient être des exemples de telles activités.

L'objectif de cette recherche est d'étudier la précision absolue et la validité des données d'altitude provenant de différentes sources pour la génération de courbes de niveaux pour des cartes topographiques à différentes échelles. De plus, l'étude s'intéresse à deux autres facteurs topographiques, à savoir la pente et les sections transversales, largement utilisés dans de nombreuses applications géomatiques. Cela constitue une préoccupation vitale dans la cartographie topographique pour déterminer si les courbes de niveaux 
et les profils topographiques basés sur les MNE open source pourraient être utilisés pour toutes les échelles de carte et les applications géomatiques. En ce qui concerne les auteurs, il pourrait s'agir de la première étude de recherche, du moins en Égypte, à aborder un sujet aussi important.

\section{Normes et Spécifications des Cartes Topographiques}

Des normes et des spécifications ont été développées dans le monde entier pour les caractéristiques des courbes de niveau, y compris la précision des données collectées et les méthodes de représentation de ces lignes importantes. Les intervalles des courbes de niveau à afficher sur une carte varient en fonction de l'échelle définie. Le terme intervalle des courbes de niveau ou équidistance représente la distance verticale ou la différence d'élévation entre deux courbes de niveau successives. L'échelle d'une carte est le rapport entre la longueur d'une ligne sur la carte et sa longueur réelle au sol. Traditionnellement, les cartes topographiques sont dessinées à des échelles cartographiques moyennes telles que 1:5000, 1:10000, 1:25000 et 1:50000. De manière générale, la sélection de l'intervalle des courbes de niveau approprié pour une carte spécifique dépend de plusieurs facteurs, notamment l'échelle de la carte, l'étendue de la zone étudiée, la nature de la topographie, le nombre et la distribution de l'ensemble de données de hauteur disponible, et le but de la carte lui-même (Survey of India, 2009). En règle générale, l'intervalle des courbes de niveau approprié sur une carte peut être estimé à trois fois l'erreur quadratique moyenne (Root Mean Square Error, RMSE) des données de hauteur d'entrée utilisées (ASPRS, American Society for Photogrammetry and Remote Sensing, 2015). La précision verticale est représentée par le RMSE des ensembles de données de hauteur utilisés selon l'équation:

$$
\text { RMSE }=\sqrt{\frac{\sum_{1}^{n}\left(P_{i}-O_{i}\right)^{2}}{n}}
$$

où $P_{i}$ et $O_{i}$ sont respectivement la hauteur prédite et observée du point $i$, et $n$ le nombre total de points.

Des exemples de normes et de spécifications de cartes topographiques, en particulier pour les caractéristiques des courbes de niveau, sont résumés ici. Les spécifications de la carte australienne indiquent que la précision d'altitude minimale des ensembles de données pour générer des courbes de niveau est de 5, 10, 25 mètres pour les échelles de carte 1:25000, 1:100000 et 1:250000 respectivement (Geoscience Australia, 2021). Au Burundi, les intervalles des courbes de niveau pour les cartes topographiques 1:5000 et 1:25000 sont respectivement de 5 et 10 mètres et la précision verticale de ces 
courbes de niveau sont respectivement inférieures à 2.5 et 5.0 mètres (JICA, Japan International Cooperation Agency, 2013). De plus, les intervalles des courbes de niveau suggérés en Inde pour les cartes 1:5000, 1:10000 1:25000 et 1:50000 sont respectivement de $1.25,5.0,8.0$ et 15 mètres (NDMA, National Disaster Management Authority, 2016). En outre, l'Institut d'études géologiques des États-Unis (United States Geological Survey, USGS) déclare que la norme de précision verticale exige que l'élévation de 90 pour cent de tous les points de hauteur utilisés soit correcte dans la moitié de l'intervalle des courbes de niveau (USGS, US Geological Survey, 1999). De plus, les spécifications cartographiques de l'État du Qatar a défini les intervalles des courbes de niveau appropriés de $2 \mathrm{~m}, 5 \mathrm{~m}$ et $10 \mathrm{~m}$ pour les échelles cartographiques 1:5000, 1:10000 et 1:200000 respectivement. De plus, le RMSE des hauteurs utilisées dans la cartographie topographique est un tiers de l'intervalle des courbes de niveau requis (Qatar GIS Center, 2009).

Au niveau national, l'Egyptian Survey Authority (ESA) a publié en 2020 les spécifications mises à jour des cartes topographiques (ESA, Egyptian Survey Authority, 2020). Pour les courbes de niveau des cartes topographiques, les normes égyptiennes stipulent que le RMSE des ensembles de données utilisés pour générer les courbes de niveau doit être inférieur à un tiers de l'intervalle des courbes de niveau. Les intervalles des courbes de niveau utilisés pour les échelles de carte 1:10000, 1:25000, 1:50000, 1:100000 et 1:250000 sont respectivement de 2, 5, 10, 20, 50 mètres. Si les courbes de niveau ne sont pas affichées aux échelles de carte 1:5000 et 1:2500, les hauteurs ponctuelles doivent être tracées sur tous les 100 et $50 \mathrm{~m}$ horizontalement avec des niveaux de précision verticale équivalents respectivement à 0.75 et 0.40 mètre. Pour conclure ce point, le tableau (1) présente des exemples typiques d'intervalle des courbes de niveau et de précision verticale utilisés dans la production de cartes topographiques avec plusieurs échelles cartographiques dans le monde entier.

Le relief, la pente et les sections transversales constituent des facteurs importants pour caractériser la topographie d'une region (Khal et al., 2020). Le relief, R, est la différence de hauteur entre les hauteurs maximale (Hmax) et minimale (Hmin) de la région. La pente entre deux points est leur différence de hauteur divisée par la distance horizontale entre eux. La pente peut être évaluée en unités de degrés ou en pourcentage selon l'équation (3):

$$
R=H_{\max }-H_{\min }
$$


Tableau 1. Normes types des cartes topographiques.

\begin{tabular}{|l|c|c|}
\hline \multicolumn{1}{|c|}{ Echelle } & $\begin{array}{c}\text { Intervalle des } \\
\text { courbes de niveau }\end{array}$ & $\begin{array}{c}\text { Précision verticale } \\
(\mathbf{m})\end{array}$ \\
\hline $1: 5000$ & 1.25 & \pm 0.4 \\
\hline $1: 10000$ & $2.0 / 5.0$ & $\pm 0.7 / \pm 1.7$ \\
\hline $1: 25000$ & $5.0 / 8.0 / 10.0$ & $\pm 1.7 / \pm 2.6 / \pm 3.3$ \\
\hline $1: 50000$ & 10.0 & \pm 3.3 \\
\hline $1: 100000$ & $10.0 / 20.0$ & $\pm 3.3 / \pm 6.7$ \\
\hline $1: 125000$ & $25.0 / 50.0$ & $\pm 8.3 / \pm 16.7$ \\
\hline
\end{tabular}

$$
S=\frac{\Delta H}{D} x 100
$$

De plus, un nouveau facteur appelé (Slope Validity, $S V$ ) est proposé, ici, pour comparer les performances des pentes estimées basées sur des MNE par rapport aux pentes précises. Il est calculé comme le pourcentage de la pente, entre deux points, à partir des deux MNE et des hauteurs précises connues à ces points de contrôle, comme suit:

$$
S V_{\text {GDE.M }}=\frac{S_{\text {GDE.M }}}{S_{\text {known }}} x 100
$$

Concernant la topographie du terrain, elle pourrait être divisée en classes selon plusieurs facteurs tels que la pente. Le tableau (2) présente un exemple d'unités topographiques basées sur les pentes (Cahyaningsih et al., 2016).

\section{Méthodologie et Source de Données}

Les modèles numériques d'élévation (MNE) utilisés, ici, incluent les données NASADEM_HGT v. 1, SRTMGL1 v. 3, ASTER v. 3 et ACE2. Ils constituent les MNT globaux les plus récents au moment de la réalisation de cette recherche. Le tableau (3) résume les caractéristiques de ces MNT sélectionnés, notamment:

1) SRTM: Shuttle Radar Topography Mission (SRTM) est un MNT global, qui a deux versions: SRTM 1 avec une résolution spatiale de 1 seconde d'arc, i.e., soit environ 30 mètres, et le modèle SRTM 3 avec une résolution de 3 secondes d'arc. SRTMGL1 v. 3 (USGS, US Geological Survey, 2021a) a été utilisé ici. (Téléchargeable gratuitement depuis, le site; https://earthexplorer.usgs.gov/). 
2) ACE2: The Altimeter Corrected Elevations (L'Altimètre Corrigé Elévations), Version 2 est un modèle d'élévation numérique global créé par fusion synergique de l'ensemble de données SRTM avec l'Altimétrie Radar Satellite dans la région délimitée par les coordonnées $60^{\circ} \mathrm{N}$ et $60^{\circ} \mathrm{S}$. ACE2 a été développé à des résolutions de 3.9 et 30 secondes d'arc et 5 minutes d'arc (Smith et Berry, 2019). La résolution de 3 secondes d'arc a été utilisée les MNE sont disponibles en téléchargement à partir du site https://sedac.ciesin.columbia.edu/data/set/dedc-ace-v2).

3) ASTER: The Advanced Spaceborne Thermal Emission and Reflection Radiometer (Le radiomètre spatial avancé d'émission et de réflexion thermique (ASTER) est un MNT global de résolution d'une seconde d'arc (US Open Data Catalouge, 2021). La version 3 d'ASTER a été rendue publique en 2019 (téléchargeable depuis le site, https://asterweb.jpl.nasa.gov/gdem.asp).

4) NASADEM_HGT v. 1: Un MNE développé par la National Aeronautics and Space Administration (NASA) des États-Unis en collaboration avec des agences allemandes et italiennes, et publié en 2020. Il est basé sur un traitement amélioré et la fusion de plusieurs sources de données telles que SRTM, ASTER, Ice, Cloud, et Land Elevation Satellite (ICESat) et autres sources (USGS, US Geological Survey, 2021b). Il peut être téléchargé à partir de https://search.earthdata.nasa.gov/.

La principale zone d'étude est la région du delta du Nil, en Égypte. Elle s'étend de la ville de Damiette à l'est jusqu'à la ville d'Alexandrie le long de la mer Méditerranée et s'étend au sud jusqu'au Caire (Figure 1a). 540 GCP avec des hauteurs précises, à \pm 0.05 mètre, ont été collectés dans le delta du Nil. Les quatre MNE ont été téléchargés, à partir de leur site Web, pour cette région. Tout d'abord, le modèle topographique de la région a été étudié en utilisant le logiciel Arc GIS 10.4.1 pour convertir les hauteurs basées sur MNE en pourcentages de pente. Il a été constaté que la pente moyenne du delta du Nil est de $3.1 \%$ (Figure 1b) ce qui indique que sa topographie pourrait être considérée comme une classe modérée ou de plateaux (Tableau 2). Par conséquent, une autre zone d'étude a été ajoutée. Elle représente la ville de La Mecque dans la région sud-ouest du Royaume d'Arabie Saoudite (KSA) à environ $70 \mathrm{~km}$ à l'est de la ville de Djeddah sur la mer Rouge (Figure 2a). $175 \mathrm{GCP}$ avec des hauteurs précises, de \pm 0.05 mètre, ont été collectés à La Mecque. De même, les quatre MNE ont été téléchargés et les pentes topographiques ont été estimées (Figure $2 b$ ). Il a été constaté que la 
pente moyenne de la Mecque est de $17.4 \%$, ce qui indique que sa topographie pourrait être considérée comme une classe élevée ou de collines.

Après avoir téléchargé les grilles spatiales des quatre MNE étudiés pour les deux zones d'étude (Figures 3 et 4), leurs hauteurs interpolées aux points de contrôle au sol (GCP) disponibles ont été comparées aux hauteurs précises connues en utilisant le Logiciel Arc GIS 10. Les résultats obtenus sont présentés et étudiés dans la section suivante.

Tableau 2. Classes topographiques basés sur la pente.

\begin{tabular}{|c|c|c|}
\hline Classe topographique & Type de pente & Pente (\%) \\
\hline Basses-terres & Douce & $<3 \%$ \\
\hline Plateaux & Modérée & $3 \%-8 \%$ \\
\hline Hautes terrasses & Modérée & $8 \%-12 \%$ \\
\hline Collines & Forte & $12 \%-40 \%$ \\
\hline Montagnes & Très forte & $>40 \%$ \\
\hline
\end{tabular}

Tableau 3. Caractéristiques des MNT globaux utilisés.

\begin{tabular}{|c|c|c|c|}
\hline \multirow{2}{*}{ MNE } & \multirow{2}{*}{ Année } & \multicolumn{2}{|c|}{ Résolution spatiale } \\
\cline { 3 - 4 } & & Seconde d'arc & Mètre \\
\hline ASTER v.3 & 2019 & $1 "$ & $\sim 30$ \\
\hline ACE2 & 2019 & $3 "$ & $\sim 90$ \\
\hline SRTMGL1 v. 3 & 2019 & $1 "$ & $\sim 30$ \\
\hline NASADEM_HGT v. 1 & 2020 & $1 "$ & $\sim 30$ \\
\hline
\end{tabular}



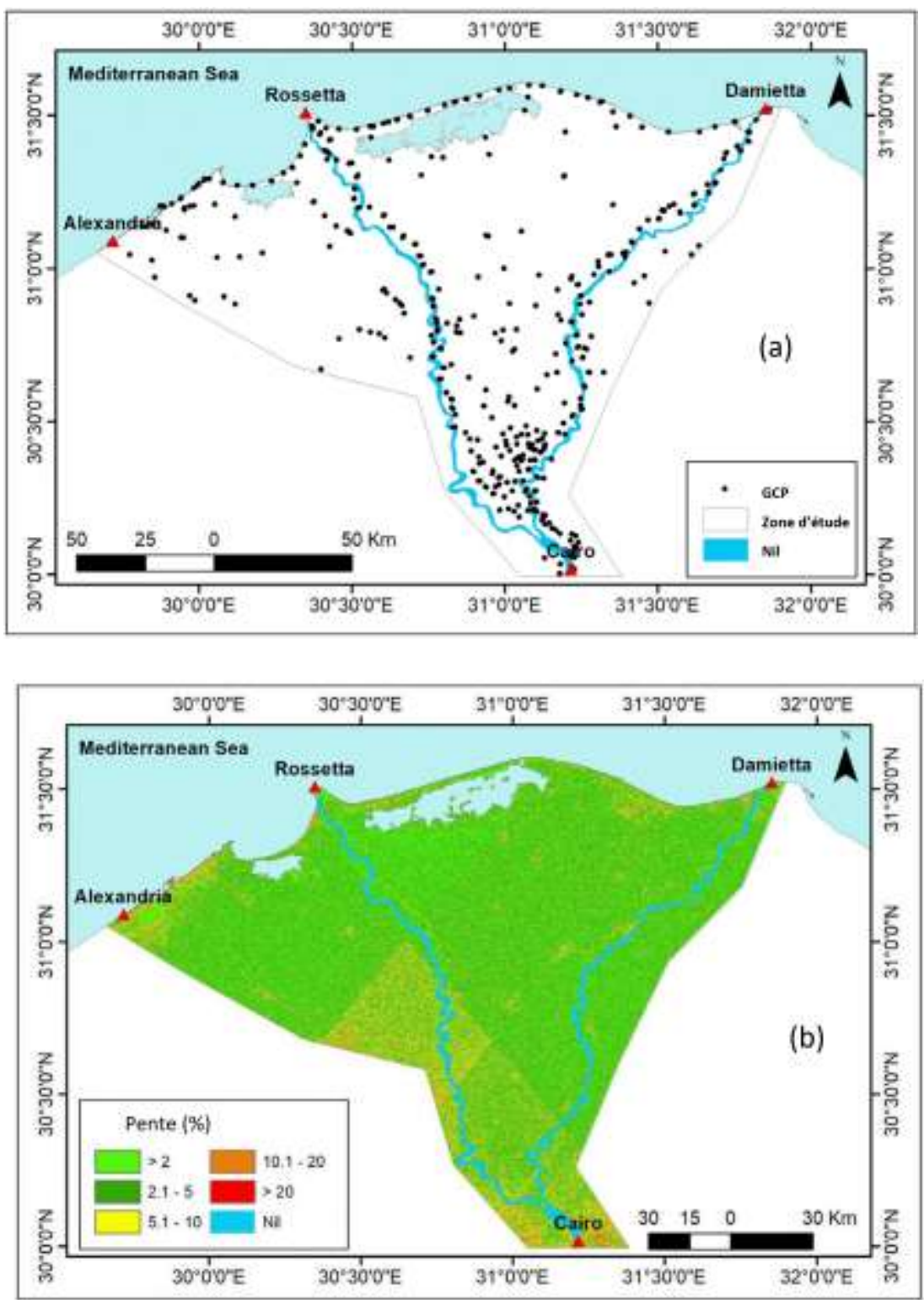

Figure 1. Zone d'étude du delta du Nil.

(a) Données disponibles; (b) Topographie. 

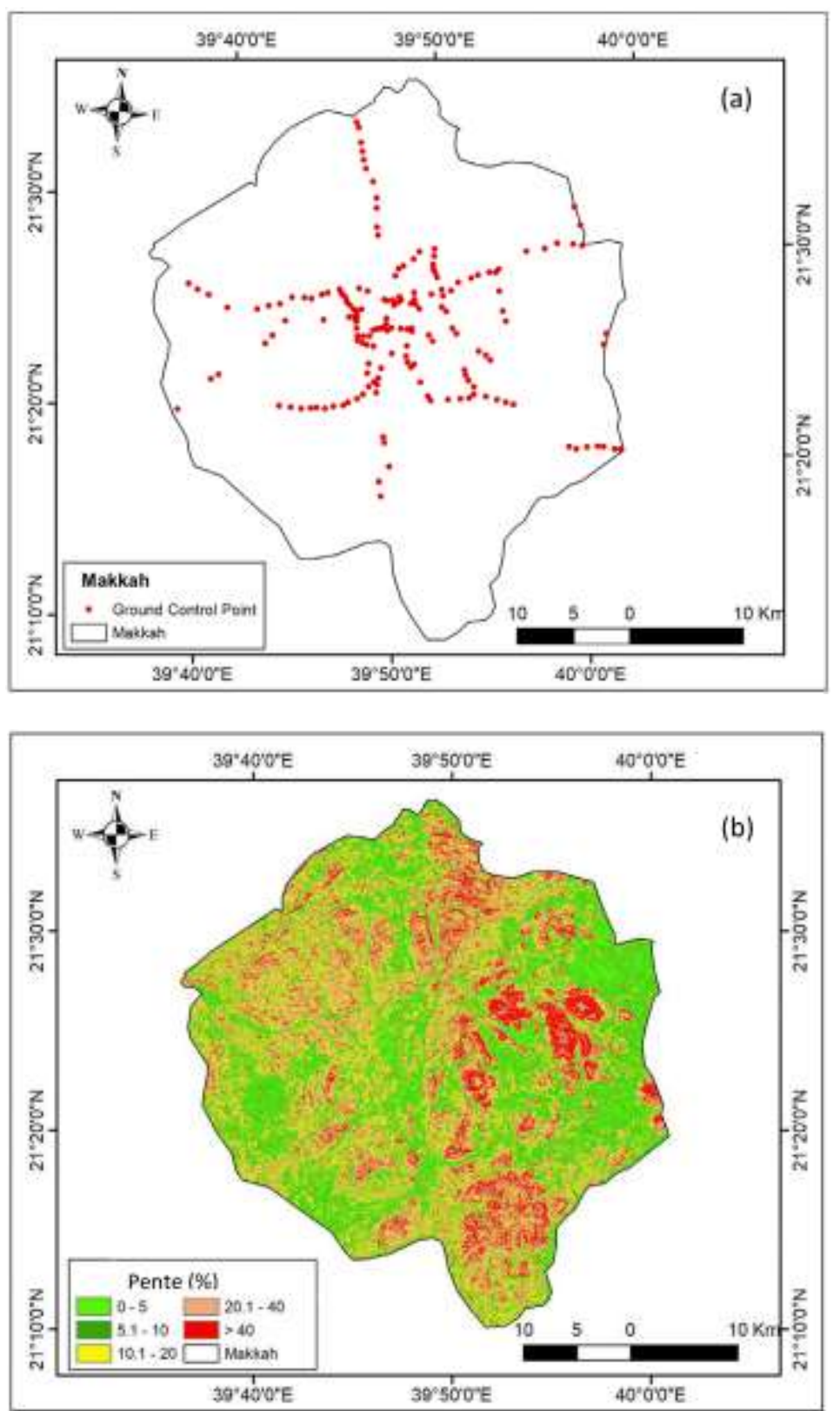

Figure 2. Zone d'étude de la Mecque.

(a) Données disponibles; (b) Topographie 

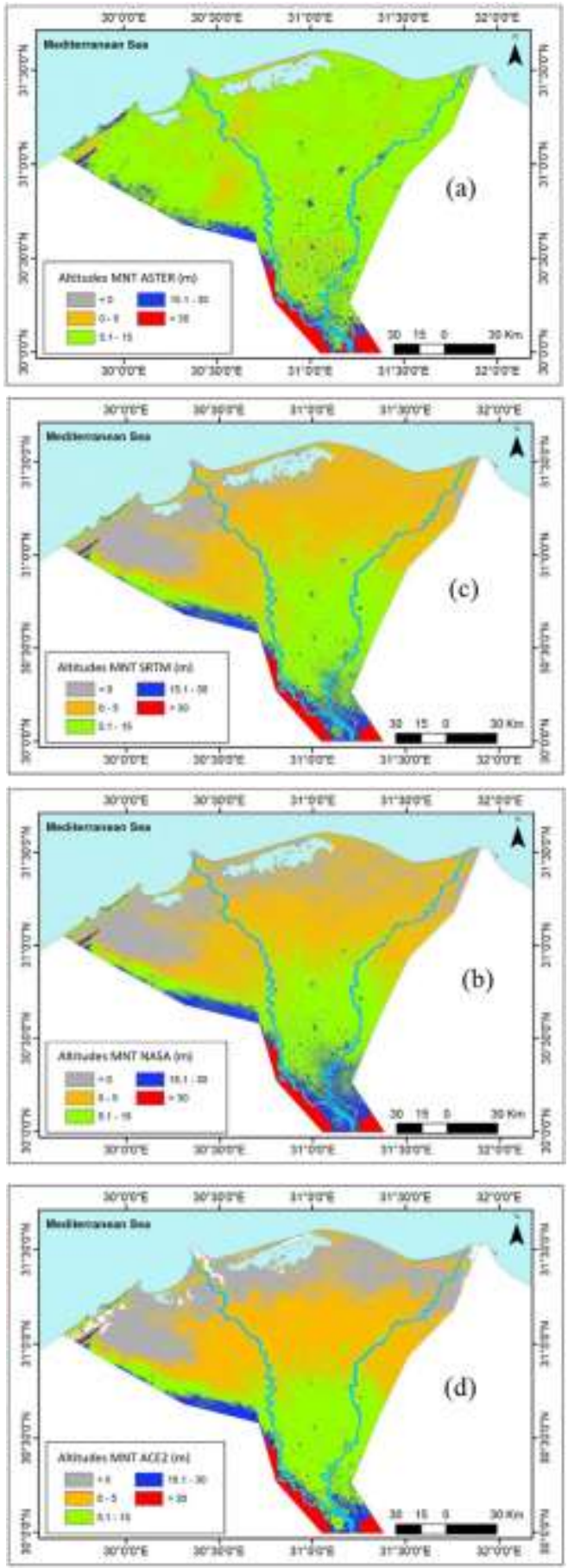

Figure 3. Topographie du delta du Nil à partir des MNE. (a) ASTER; (b) NASADEM; (c) SRTM; (d) ACE2. 

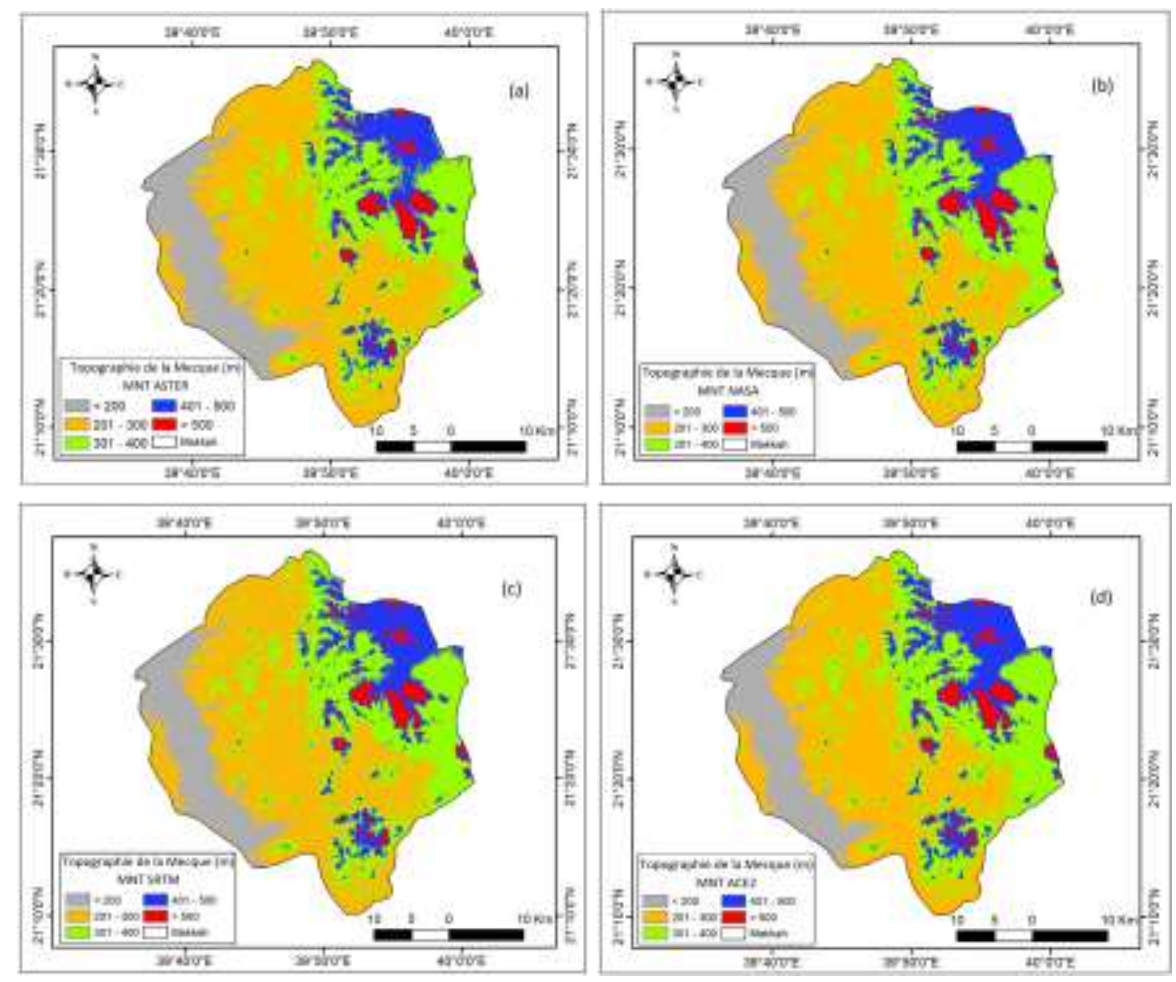

Figure 4. Topographie de La Mecque à partir des MNE.

(a) ASTER; (b) la NASA; (c) SRTM; (d) AEC2.

\section{Résultats et Discussion}

Le tableau (4) présente les statistiques des hauteurs observées et basées sur le MNE dans la région du delta du Nil. De manière générale, on peut remarquer que la hauteur moyenne et le relief (équation (2)) du seul MNE ASTER ne sont pas proches de ceux des hauteurs observées, tandis que les trois autres MNE ont produit des estimations relativement comparables. Ensuite, les erreurs des MNE ont été comparées aux hauteurs connues des GCP (Tableau 5). On peut remarquer que le RMSE (Équation (1)) des modèles AEC2, STRMGL1 et NASADEM est égal à \pm 2.5 mètres environ, tandis que le modèle ASTER produit le RMSE le plus élevé ( \pm 5.1 mètres). Concernant l'erreur de hauteur moyenne, on peut voir que le MNT NASA produit la plus petite valeur de 1.8 mètre par rapport aux trois autres modèles. La distribution des erreurs a été tracée sur la figure (5) pour tous les MNE, ce qui met l'accent sur les performances globales des MNE étudiés. On peut se rendre compte à partir de cette figure (5) que les performances du modèle ASTER sont loin de celles des autres modèles. 
Tableau 5. Statistiques des erreurs d'altitude des MNE dans la zone du delta du Nil.

\begin{tabular}{|c|c|c|c|c|c|}
\hline & Minimum & Maximum & Variation & Moyenne & RMSE \\
\hline ASTER v.3 & -19.901 & 18.895 & 38.796 & -2.148 & \pm 5.108 \\
\hline ACE2 & -11.518 & 9.248 & 20.766 & 2.288 & \pm 2.618 \\
\hline SRTM & -6.320 & 9.500 & 15.820 & 1.971 & \pm 2.584 \\
\hline NASA HGT & -5.438 & 9.243 & 14.681 & 1.756 & \pm 2.521 \\
\hline
\end{tabular}

En comparant les valeurs RMSE des MNE étudiés (Tableau 5) avec la précision verticale requise des ensembles de données utilisés (Tableau 1), on peut conclure (Tableau 6) que tous les MNE ne sont pas adaptés pour développer des intervalles des courbes de niveau inférieurs à 5 mètres. Cela signifie que de tels modèles ne doivent pas être utilisés pour la cartographie topographique à l'échelle 1:25000 ou plus. Pour les cartes à l'échelle 1:50000 et 1:100000 avec des intervalles des courbes de niveau de 8 ou 10 mètres, on constate que le modèle ASTER doit être évité alors que les trois autres modèles répondent aux spécifications requises. De plus, il est clair que pour les échelles cartographiques de 1:250000 ou moins, tous les MNE étudiés pourraient être utilisés.

Tableau 6. Validité du MNE pour la cartographie topographique dans la zone du delta du Nil.

\begin{tabular}{|c|c|c|}
\hline Echelle & $\begin{array}{c}\text { Intervalles des } \\
\text { courbes de niveau }\end{array}$ & MNE Valid \\
\hline $1: 5000$ & 1.25 & \multirow{3}{*}{ NA } \\
\hline $1: 10000$ & $2.0 / 5.0$ & \\
\hline $1: 25000$ & 5.0 & \\
\hline $1: 50000$ & $8.0 / 10.0$ & ACE2, SRTMGL1 v.3, NASADEM_HGT v.1 \\
\hline \multirow{2}{*}{$1: 100000$} & 10.0 & \multirow{2}{*}{ ASTER v.3, ACE2, SRTMGL1 v.3, } \\
\hline & 20.0 & \\
\hline \multirow{2}{*}{$1: 250000$} & 25.0 & \multirow{2}{*}{ NASADEM_HGT v.1 } \\
\hline & 50.0 & \\
\hline
\end{tabular}

Comme mentionné précédemment, la région du delta du Nil représente une zone plate en termes de topographie. Par conséquent, les mêmes étapes ont été effectuées pour étudier les performances des MNE sur la ville de La 
Mecque en tant que région à topographie modérée. Le tableau (7), le tableau (8) et la figure (6) présentent les résultats obtenus sur la base de l'ensemble des données disponibles. On peut remarquer à partir du tableau (7) que seul le modèle SRTMGL1 a produit des estimations de relief loin de la précision observée. Pour l'erreur de hauteur moyenne, seul le modèle ACE2 diffère un peu des trois autres MNE. Cependant, les résultats des différences de hauteur par rapport aux GCP connus (Tableau 8) sont plus importants pour analyser la précision des MNE. On peut se rendre compte que les MNT NASA et SRTMGL ont produit les plus petites valeurs RMSE, tandis que le modèle ASTER donne la plus grande. En comparant les histogrammes d'erreur sur les deux zones d'étude (Figures 5 et 6), on peut remarquer que la performance des MNE étudiés est plus précise sur la région plate du delta du Nil que celle à topographie vallonnée de la ville de La Mecque. Cette remarque est également valable lorsque l'on compare les valeurs RMSE des MNE dans les deux régions. Dans le delta du Nil, le RMSE varie de \pm 2.5 à \pm 5.0 mètres, tandis qu'à la Mecque le RMSE varie entre \pm 5.2 et \pm 8.0 mètres environ. Par conséquent, on peut conclure que la précision des MNE étudiés est plus faible dans les régions à topographie modérée que dans les zones plates.

Tableau 7. Statistiques des sources de hauteur dans la zone de la Mecque.

\begin{tabular}{|c|c|c|c|c|c|}
\hline & Minimum & Maximum & Relief & Moyenne & RMSE \\
\hline $\begin{array}{c}\text { Hauteurs } \\
\text { observées }\end{array}$ & 147.135 & 423.187 & 274.823 & 272.146 & \pm 59.189 \\
\hline ASTER v.3 & 144.185 & 419.008 & 274.823 & 272.146 & \pm 59.189 \\
\hline ACE2 & 150.695 & 422.845 & 272.150 & 278.933 & \pm 60.068 \\
\hline SRTM & 140.482 & 424.860 & 284.378 & 276.082 & \pm 59.541 \\
\hline NASA HGT & 148.267 & 419.924 & 271.657 & 273.295 & \pm 59.005 \\
\hline
\end{tabular}

Tableau 8. Statistiques des erreurs d'altitude des MNE dans la zone de la Mecque.

\begin{tabular}{|c|c|c|c|c|c|}
\hline & Minimum & Maximum & Variation & Moyenne & RMSE \\
\hline ASTER v.3 & -18.456 & 22.830 & 41.286 & -0.334 & \pm 7.996 \\
\hline ACE2 & -34.463 & 5.948 & 40.412 & -7.121 & \pm 6.675 \\
\hline SRTM & -16.680 & 7.820 & 24.500 & -4.270 & \pm 5.161 \\
\hline NASA HGT & -13.839 & 11.219 & 25.058 & -1.483 & \pm 5.243 \\
\hline
\end{tabular}




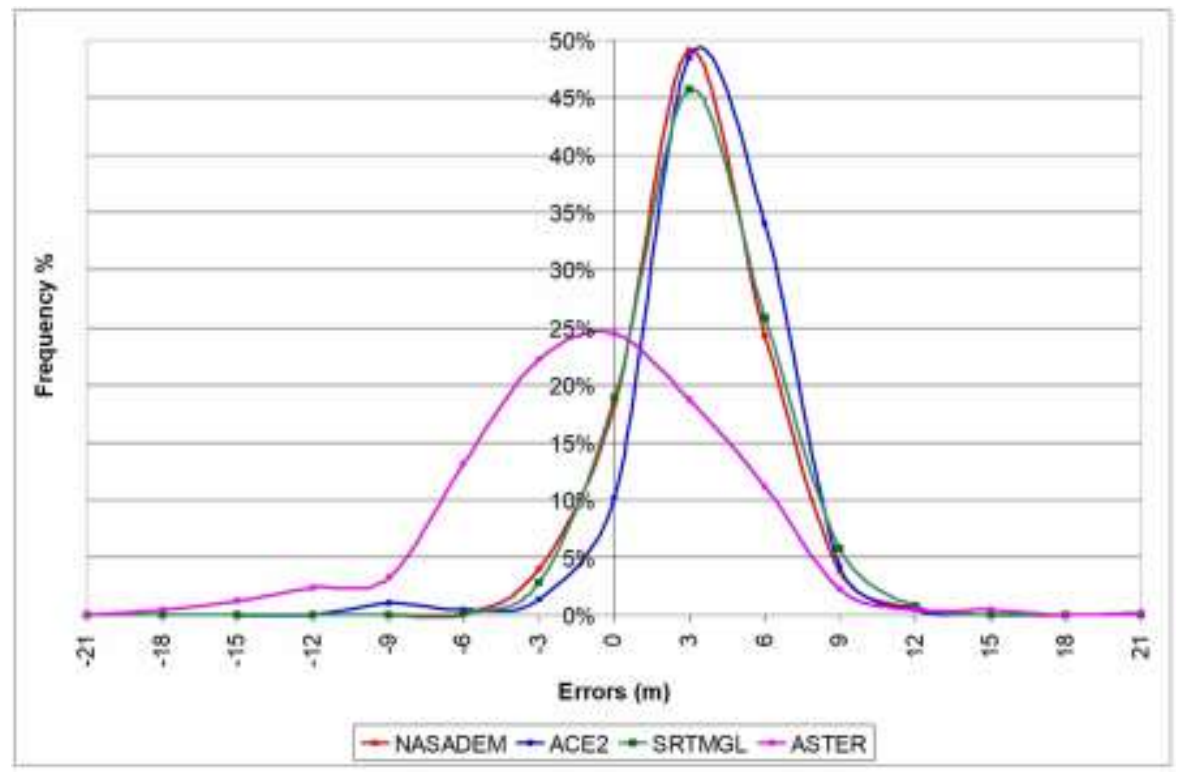

Figure 5. Histogrammes des erreurs de hauteur des MNE dans la région du Delta du Nil.

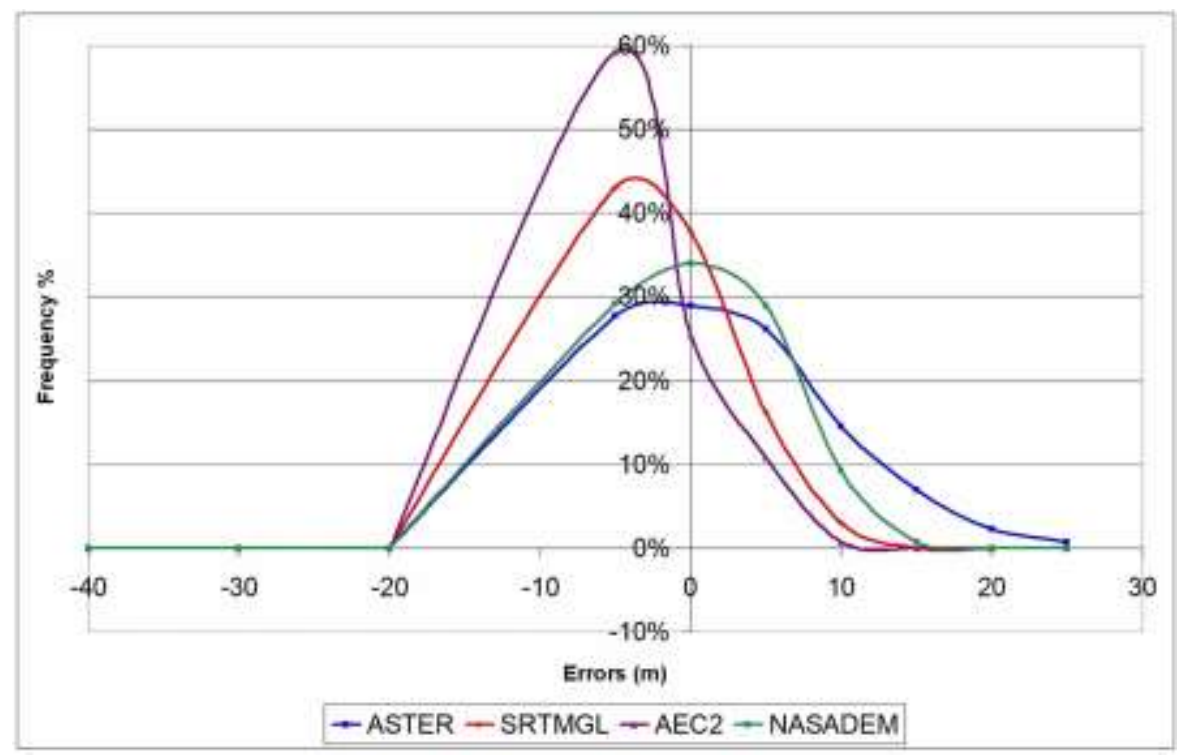

Figure 6. Histogrammes des erreurs de hauteur des MNE dans la région de La Mecque. 
Une comparaison a été également effectuée entre les valeurs RMSE des MNE étudiés à La Mecque (Tableau 8) avec la précision verticale de 1'ensembles des données utilisées (Tableau 1). On peut voir dans le tableau (9) que tous les MNE ne sont pas adaptés pour développer des intervalles des courbes de niveau inférieurs à 5 mètres. Cela signifie que ces modèles ne conviennent pas à la cartographie topographique à l'échelle 1:50000 ou plus avec des intervalles des courbes de niveau égaux ou inférieurs à 10 mètres. Pour une carte à l'échelle 1:100000 avec des intervalles des courbes de niveau de 20 mètres, on peut voir que le modèle ASTER doit être évité tandis que les trois autres modèles répondent aux spécifications requises. À partir de la figure (6), on peut voir qu'ASTER a produit le modèle de fréquence d'erreurs le moins précis sur les points de contrôle par rapport aux autres MNE. De plus, il est clair que pour les échelles cartographiques de 1:250000 ou moins avec des intervalles des courbes de niveau de plus de 25 mètres, tous les MNE étudiés pourraient être utilisés. De plus, on peut conclure d'après les tableaux (6) et (9) que l'utilisation des MNE dans la cartographie topographique diffère considérablement entre les régions plates et vallonnées.

Tableau 9. Validité du MNE pour la cartographie topographique dans la zone de la Mecque.

\begin{tabular}{|l|c|c|}
\hline \multicolumn{1}{|c|}{ Echelle } & $\begin{array}{c}\text { L'intervalle des } \\
\text { courbes de niveau (m) }\end{array}$ & MNE valide \\
\hline $1: 5000$ & 1.25 & \multirow{2}{*}{ NA } \\
\hline $1: 10000$ & $2.0 / 5.0$ & \\
\hline $1: 25000$ & 5.0 & ACE2, SRTMGL1 v.3, NASADEM_HGT \\
$1: 50000$ & $8.0 / 10.0$ & v.1 \\
\hline \multirow{2}{*}{$1: 100000$} & 10.0 & ASTER v.3, ACE2, SRTMGL1 v.3, \\
& 20.0 & NASADEM_HGT v.1 \\
\hline \multirow{2}{*}{$1: 250000$} & 50.0 & \\
\hline
\end{tabular}

Pour analyser les pentes estimées basées sur le MNE (équation (3)) et les profils topographiques, deux coupes transversales ont été réalisées dans la ville de La Mecque sur la base des GCP disponibles. La première (B1-B2) s'étend d'ouest en est en passant par $28 \mathrm{GCP}$, tandis que l'autre coupe transversale (A1-A2) s'étend du nord au sud le long de 27 GCP (Figure 7). La longueur des deux sections transversales est d'environ 35 kilomètres. Pour étudier la validité de l'estimation de pente basée sur le MNE le long d'une section transversale, chacun est divisé par des intervalles de 5 kilomètres. L'équation (4) a été appliquée pour calculer le facteur de validité de pente (SV) à chaque intervalle pour les deux sections. La figure 8 
présente les résultats obtenus, ce qui révèle deux remarques importantes. Premièrement, toutes les pentes basées sur MNE ne correspondent pas aux pentes réelles du GCP connu sur la longueur de la section transversale jusqu'à 25 et 30 kilomètres pour les deux profils respectivement.

Deuxièmement, les pentes basées sur les MNE pour les profils de plus de 30 kilomètres de longueur sont proches des pentes précises dans une valeur de $\pm 10 \%$ environ. Par conséquent, on peut conclure que les MNE ne devraient pas être utilisés pour développer des sections transversales topographiques inférieures à 30 kilomètres. De plus, on peut dire que les profils topographiques basés sur les MNE ne doivent pas être utilisés dans des applications d'ingénierie et de géomatique qui nécessitent des informations précises sur les hauteurs et les pentes. Par conséquent, les résultats obtenus de la présente étude, basés sur les ensembles de données disponibles, ont montré que les MNE ne sont pas des sources d'altitudes appropriées pour la cartographie topographique à des échelles cartographiques moyennes et grandes, et ne peuvent pas être utilisés pour un profilage topographique précis dans l'ingénierie et pour des applications géomatiques précises.

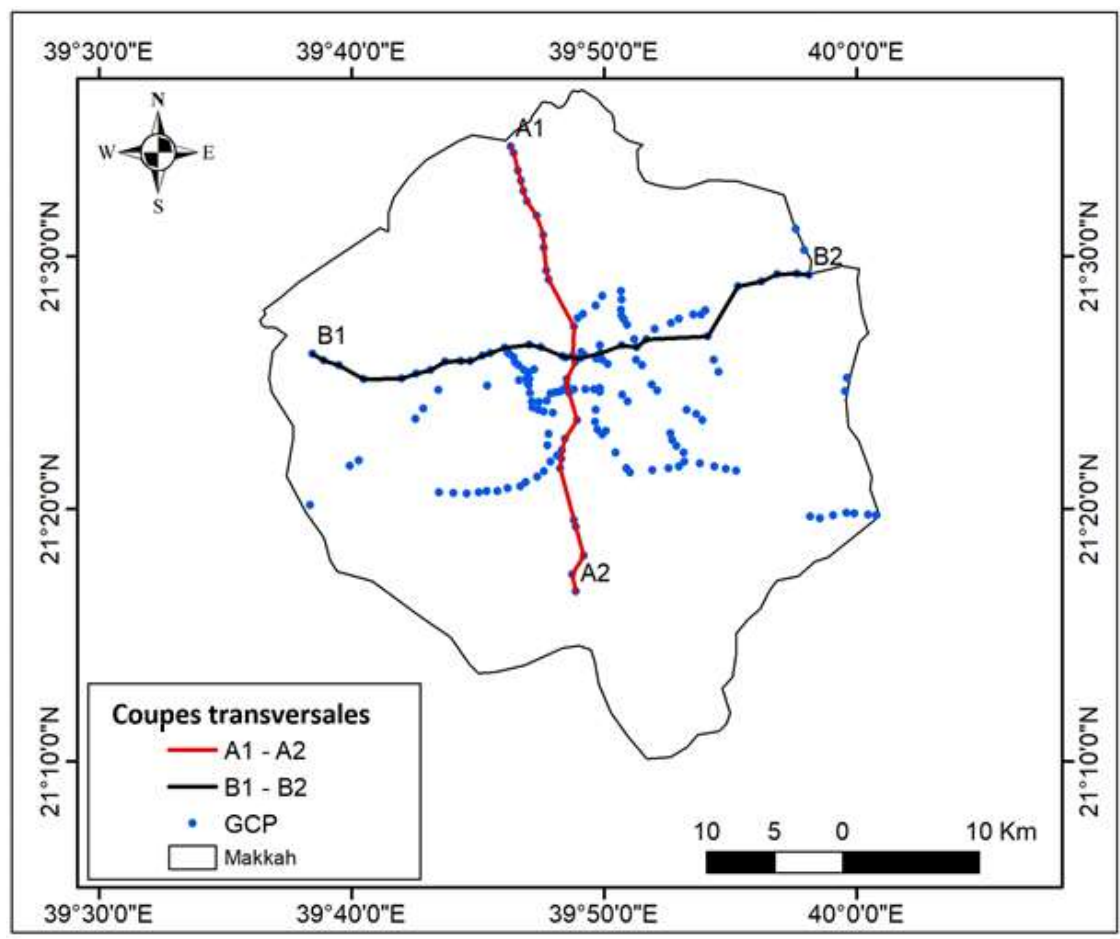

Figure 7. Coupes transversales dans la région de La Mecque. 

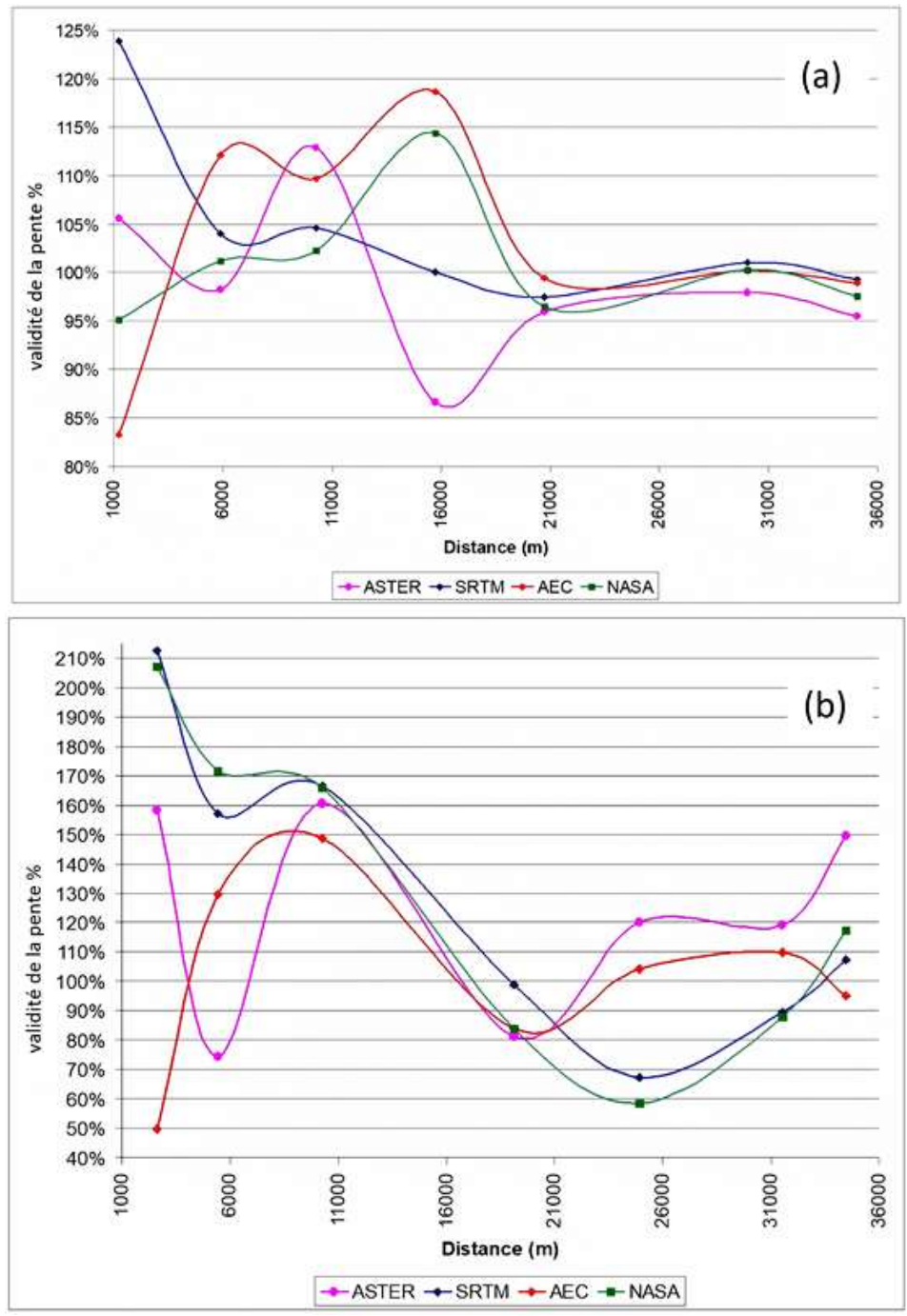

Figure 8. Validité de la pente sur des coupes transversales dans la région de La Mecque.

\section{Conclusions}

Les MNE open-source ont été utilisés au cours des deux dernières décennies pour la cartographie topographique et plusieurs activités géomatiques. Les spécifications des cartes topographiques reposent sur l'intervalle des courbes de niveau approprié pour chaque échelle de carte et sur la précision des informations sur les hauteurs utilisées. Des exemples de 
normes de cartographie topographique de plusieurs pays ont été collectés et étudiés. La présente étude vise à examiner la validité et la fiabilité des altitudes basées sur les MNE pour la cartographie topographique à plusieurs échelles cartographiques. En particulier, elle étudie les performances des MNE pour les intervalles des courbes de niveau, l'estimation de la pente et la génération de profils topographiques nécessaires pour les applications d'ingénierie et environnementales en Égypte et en Arabie Saoudite.

Deux zones d'étude ont été utilisées pour étudier les performances de quatre MNE pour la cartographie topographique sur des régions topographie plate et vallonnée. Sur la base des ensembles de données disponibles dans les deux zones d'étude, plusieurs résultats importants ont été obtenus. En utilisant des points de contrôle connus, il a été constaté que la précision des MNE étudiés varie de \pm 2.5 à \pm 5.1 mètres dans la région du delta du Nil, alors qu'elle varie entre \pm 5.1 et \pm 8.0 mètres dans la région de La Mecque. Cela indique que l'utilisation des MNE dans la cartographie topographique diffère considérablement entre les régions plates et vallonnées. Sur la base de l'intervalle des courbes de niveau requis sur plusieurs échelles de cartes topographiques, il a été constaté que les MNE ne sont pas appropriés pour générer un intervalle des courbes de niveau inférieur à 10 mètres. Par conséquent, il est suggéré de ne pas utiliser les MNE pour réaliser des cartes topographiques à l'échelle 1:25000 ou plus dans les régions plates et à l'échelle 1:50000 ou plus dans les régions vallonnées.

En termes d'estimation de pente et de développement d'un profil topographique, les résultats obtenus ont révélé que toutes les pentes basées sur le MNE ne correspondent pas aux pentes réelles des GCP connus sur des longueurs de section transversale allant jusqu'à 30 kilomètres. Il a également été observé que les pentes basées sur le MNE pour les profils de plus de 30 kilomètres de longueur sont proches des pentes précises dans une valeur de $\pm 10 \%$ environ. En conséquence, on pourrait conclure que les MNE ne sont pas les sources d'altitudes appropriées pour la cartographie topographique à des échelles cartographiques moyennes et grandes, et ne pourraient pas être utilisés pour le profilage topographique dans des applications précises d'ingénierie et de géomatique.

\section{Les conflits d'intérêts}

Les auteurs ne déclarent aucun conflit d'intérêt concernant la publication de cet article. 


\section{Références}

1. Abdel-Aziz, T., Dawod, G. \& Ebaid, H., 2020, "DEMs and Reliable Sea Level Rise Risk Monitoring in Nile Delta, Egypt", Discover Sustainability, 1, Article no. 6 . https://doi.org/10.1007/s43621-020-00006-7

2. Al-Karagy, E. \& Dawod, G. 2021, "Optimum Combinations of GGM and DEM Models for Precise National Geoid Development", Proceedings of Engineering and Technology Innovation, vol. 18, pp. 15-24

3. ASPRS (American Society for Photogrammetry and Remote Sensing), 2015, "ASPRS Positional Accuracy Standards for Digital Geospatial Data", Edition 1, Version 1. Photogrammetric Engineering and Remote Sensing, 81, A1-A26. https://doi.org/10.14358/PERS.81.3.A1-A26

4. Boulton, S.J. \& Stokes, M. 2018, "Which DEM Is Best for Analyzing Fluvial Land- scape Development in Mountainous Terrains?", Geomorphology, vol. 310, pp. 168-187.

5.Cahyaningsih, C., Crensonni, P., Suryadi, A., Kausarian, H., Choanji, T., Yuskar, Y. \& Putra, D. 2019, "Geomorphology and Structural Geology Characterization of Landslide Prone Area in Riau-West of Sumatra Highway", IOP Conference Series Materials Science and Engineering, 536, Article ID: 012063;

https://doi.org/10.1088/1757-899X/536/1/012063

6. Church, J., Clark, P., Cazenave, A., Gregory, J, Jevrejeva, S., Levermann, A., Merri- field, M., Milne, G., Nerem, R., Nunn, P., Payne, A., Pfeffer, W., Stammer, D. \& Unnikrishnan, A. 2013, "Sea Level Change", In: Climate Change 2013: The Physical Science Basis. Contribution of Working Group I to the Fifth Assessment Report of the Intergovernmental Panel on Climate Change (IPCC), Cambridge University Press, Cambridge, pp. 1137-1216.

7. Dawod, G. \& Al-Ghamdi, K. 2017, "Reliability of Recent Global Digital Elevation Models for Geomatics Applications in Egypt and Saudi Arabia”, Journal of Geographic Information System, vol. 9, no.6, pp. 685-698. https://doi.org/10.4236/jgis.2017.96043

8. ESA (Egyptian Survey Authority), 2020, "Technical Specifications of Surveyingand Mapping”: Part 2: Topographic Maps. A Technical Report, Giza.

9. Geoscience Australia, 2021, "National Topographic Database Structure and Specifi- cations", http://www.ga.gov.au/mapspecs/topographic/v6/section1.html\#MinimumPl animetr icElevationAccuracies

10. Hasan, E., Khan, S. \& Hong, Y. 2015, "Investigation of Potential Sea Level Rise Impact on the Nile Delta, Egypt Using Digital Elevation Models", Environmental Monitoring and Assessment, 187, 649. https://doi.org/10.1007/s10661-015-4868-9

11. Hu, Z., Peng, J., Hou, Y. \& Shan, J. 2017, "Evaluation of Recently Released Open Global Digital Elevation Models of Hubei, China", Remote Sensing, 9, no.3, 262.

https://doi.org/10.3390/rs9030262 
12. JICA (Japan International Cooperation Agency), 2013, "The Study on Establishing Digital Topographic Database for Bujumbura City, Burundi”, A Technical Report. https://openjicareport.jica.go.jp/pdf/12121588_01.pdf

13. Khal, M., Algouti, A., Algouti, A., Akdim, N., Stankevich, S. \& Menenti, M. 2020, "Evaluation of Open Digital Elevation Models: Estimation of Topographic Indices Relevant to Erosion Risk in the Wadi M'Goun Watershed, Morocco", Geosciences, vol. 6, issue 2, pp. 231-257.

https://doi.org/10.3934/geosci.2020014

14.Kulp, S. \& Strauss, 2016, "Global DEM Errors Underpredict Coastal Vulnerability to Sea Level Rise and Flooding", Frontiers in Earth Science.

https://doi.org/10.3389/feart.2016.00036

15. Mokarram, M. \& Hojati, M. 2016, "Comparison of Landform Classifications of Elevation, Slope, Relief and Curvature with Topographic Position Index in the South of Bojnoord", Ecopersia, 2, 1343-1357.

https://doi.org/10.18869/modares.ecopersia.4.2.1343

16. NDMA (National Disaster Management Authority), 2016, "National Technical Document Fir Establishing Cartographic Database in India: Generation of Large Scale (1:10,000 and 1:2,000 and Leaser) Maps for Disaster Management and Planning", A Technical Report. http://www.ndma.gov.in/sites/default/files/PDF/Technical\%20Documents/nationaltechnical-document-for-establishing-cartographic-base-in-india.pdf

17. Ogania, J., Puno, G., Alivio, M. \& Taylaran, J. 2019, "Effect of Digital Elevation Model's Resolution in Producing Flood Hazard Maps", Global Journal of Environmental Science and Management, vol. 5, no. 1, pp. 95-106.

18. Ouerghi, S., ELsheikh, R., Achour, H. \& Bouazi, S. 2015, "Evaluation and Validation of Recent Freely-Available ASTER-GDEM V.2, SRTM V.4.1 and the DEM Derived from Topographical Map over SW Grombalia (Test Area) in North East of Tunisia", Journal of Geographic Information System, vol. 7, no. 3, pp. 266-279. https://doi.org/10.4236/jgis.2015.73021

19. Patel, A., Katiyar, S. \& Prasad, V. 2016, "Performances Evaluation of Different Open Source DEM Using Differential Global Positioning System (DGPS)", The Egyptian Journal of Remote Sensing and Space Sciences, vol. 19, issue 1, pp. 7-16. https://doi.org/10.1016/j.ejrs.2015.12.004

20. Qatar GIS Center, 2009, "Qatar Survey Manual”, Version 1. A Technical Report, Qatar.

21. Sande, B., Lansen, J. \& Hoyng, C., 2012, "Sensitivity of Coastal Flood Risk Assessments to Digital Elevation Models", Water 4, no. 3, pp. 568-579. https://doi.org/10.3390/w4030568

22. Seleem, T. 2013, "Analysis and Tectonic Implication of DEM-Derived Structural Lineaments, Sinai Peninsula, Egypt”, International Journal of Geosciences, vol.4, no.1, pp.183-201. https://doi.org/10.4236/ijg.2013.41016

23. Smith, R. and Berry, P., 2019, "Altimeter Corrected Elevations", Version 2. https://sedac.ciesin.columbia.edu/data/set/dedc-ace-v2 24. Survey of India, 2009, Handbook of Topography, http://www.iism.nic.in/Documents/soichapter-xi.pdf 
25. US Open Data Catalouge, 2021, “ASTER Digital Elevation Model”, V003. https://catalog.data.gov/dataset/aster-digital-elevation-model-v003

26. USGS (US Geological Survey), 1999, "Map Accuracy Standards: Fact Sheet”, FS-171-99. A Technical Report. https://pubs.usgs.gov/fs/1999/0171/report.pdf

27.USGS (US Geological Survey) (2021a) SRTMGL1 V003, https://lpdaac.usgs.gov/products/srtmgl1v003

28.USGS (US Geological Survey) (2021b). NASADEM_HGT: Merged DEM Global 1, Arc Second v. 1.

https://lpdaac.usgs.gov/products/nasadem_hgtv001

29.Zhang, J. \& Chu, X., 2015, "Impact of DEM Resolution on Puddle Characteriza- tion: Comparison of Different Surfaces and Methods", Water 7, no. 5, pp. 2293-2313.

https://doi.org/10.3390/w7052293 


$$
\begin{aligned}
& \text { صلاحية بيانات الارتفاعات للمصادر المفتوحة لمختلف مقاييس الخرائط } \\
& \text { الطبوغرافية وتطبيقات الجيوماتكس لماتف مقات }
\end{aligned}
$$

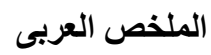

تعرض هذه الورقة نحليلا لأربعة نماذج عالمية للارتفاعات الرقمية مفتوحة المصدر ومقارنتها

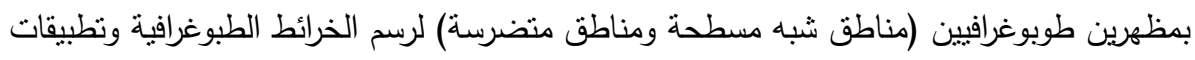

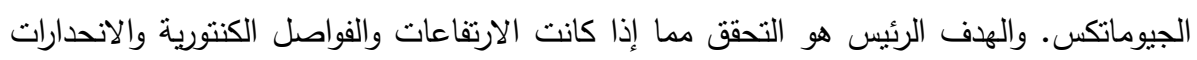

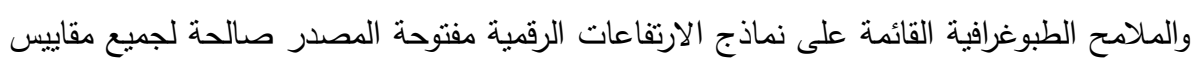

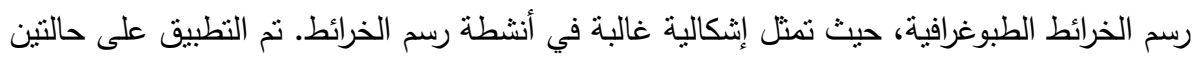

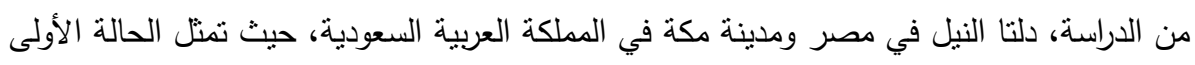
منطقة مسطحة التضاريس والثانية مثال للمناطق المتضرسة. ونماذج الارتفاع الرقمية العالمية المستخدمة في هذه الدراسة تشمل أحدث النماذج:ASTER

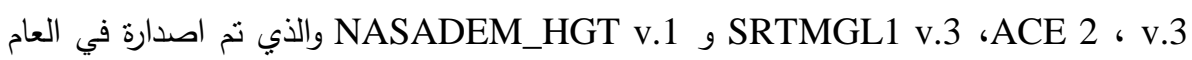

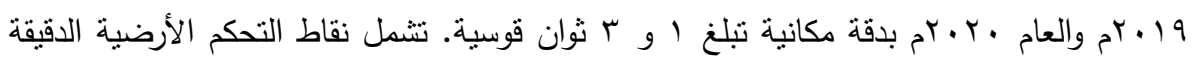

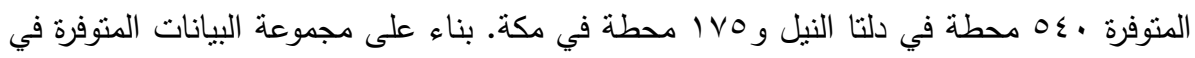
منطقتي الدراسة فقد وجد ان دقة نماذج الارتفاعات الرقمية العالمية المدروسة في نقاط التحكية التحم السابقة

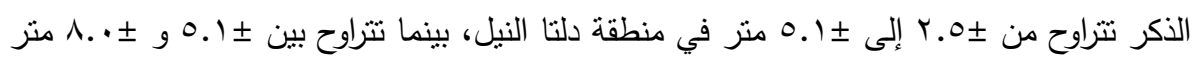

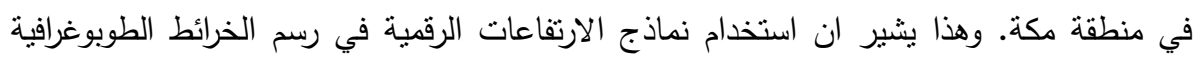

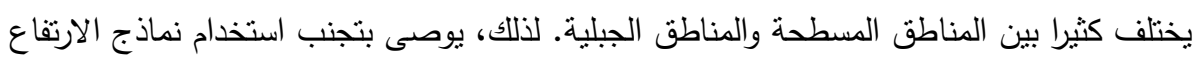

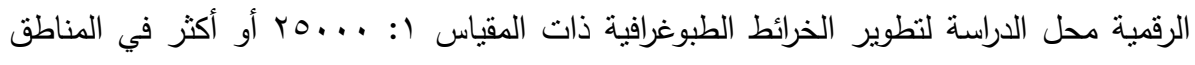
المسطحة والمقياس ا: ... . . أو أكثر في المناطق الجبلية. بالإضافة إلى ذللك، أظهرت نتائج

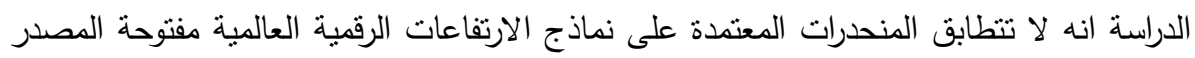

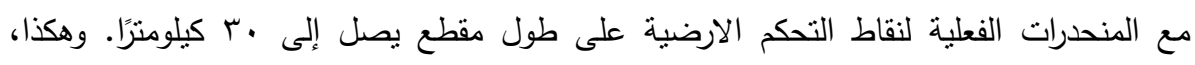

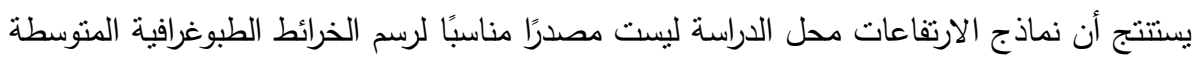
والكبيرة الحجم، ولا ينبغي استخدامها للتتميط الطبوغرافي في التطبيقات الهندسية والجيومانية الدقيقة. 